\title{
Available Bandwidth Estimation for Network Selection in Heterogeneous Environment of WLAN and WiMAX
}

\author{
Kiran Ahuja \\ Department of Electronics and \\ Communication Engineering, \\ DAV Institute of Engg. \& Tech. \\ Jalandhar, Punjab, India.
}

\author{
Brahmjit Singh \\ Professor, Department of Electronics \\ and Communication Engineering \\ National Institute of Technology \\ Kurukshetra, Haryana, India
}

\author{
Rajesh Khanna \\ Professor, Electronics and \\ Communication Engineering \\ Department, Thapar, Patiala \\ Punjab, India.
}

\begin{abstract}
:
Always best connected services (ABC) allow multimode mobile terminals to stay connected to the best available networks, at anytime according to user preferences. One of the key aspects in realizing such $\mathrm{ABC}$ service is mainly attributed to an effective and dynamic access network selection process. However, most of the previous works consider the access network selection process as a static optimization problem which fails to address the dynamic QoS conditions intrinsic in wireless networks. One of the main challenges remaining is to find an efficient way of obtaining dynamic QoS parameters such as available bandwidth. In this paper, propose a novel dynamic access network selection algorithm capable of adapting to prevailing network conditions. Proposed algorithm is an estimation process where network selection in heterogeneous wireless environment (WiMAX \& WLAN) performed using available bandwidth estimation relies on dynamic parameters designed using .net.

Keywords:
\end{abstract}

WLAN, WiMAX, Heterogeneous wireless networks, Available bandwidth

\section{INTRODUCTION:}

ABC networks and services have gained much attention recently and have been adopted as one of the main drivers of $4 \mathrm{G}$ evolution. Together with the demands of ubiquitous connectivity and high data rates in $4 \mathrm{G}$ networks, the importance of interworking between existing and possibly new radio access technologies (RAT) are prevalent since the available resources of any single network is insufficient to meet such requirements. In a nutshell, $4 \mathrm{G}$ has been envisaged as a congregation of complementary heterogeneous RATs where seamless services could be provisioned dynamically through the most efficient network based on user preferences and prevailing network conditions [1].

Further, 4G would converge into an all-IP network for integrating different RATs [2]. With the introduction of new RAT such as worldwide interoperability for microwave access (WiMAX) in the near future, we consider a heterogeneous IPbased $4 \mathrm{G}$ architecture comprising of WLAN and WiMAX as illustrated in Figure 1.

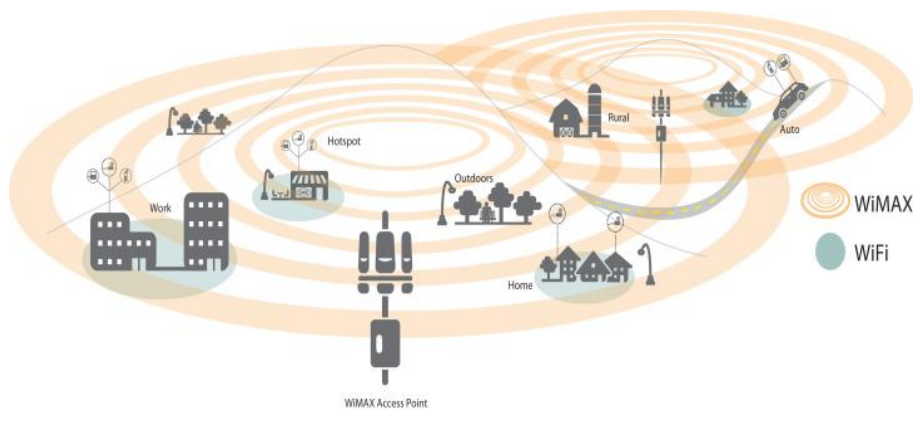

Figure 1: Using Handheld Devices in Mobile, Portable and Public Hotspot Environments [2]

The inter-working capabilities between WiMAX and WLAN enable service providers to deliver consistent, transparent, and user-friendly broadband services to their subscribers. (See Figure 2) Achieving this transparency requires two key elements: Multi-mode subscriber devices that can communicate on both WiMAX and WLAN networks and the ability to provide service across WiMAX and WLAN networks when users move between them. This is generally implemented through a controlling Access Service Network Gateway (ASN GW) and common Authentication, Authorization, and Accounting (AAA) service functionality located in the service provider network.

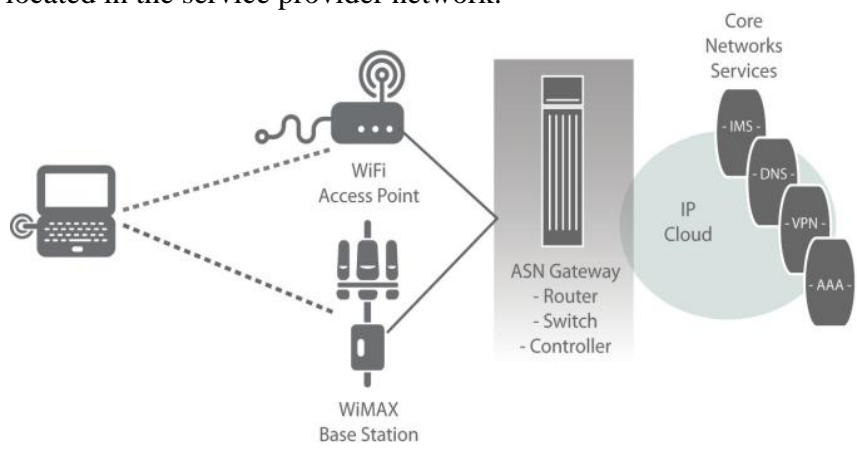

Figure 2: WiMAX/WLAN Inter-working environment [2]

The IP-based core network enables easy exploitation of existing mobile IP techniques to achieve seamless handover with QoS support [3]. However, the access network heterogeneity demands an efficient network selection scheme such that users can be best connected through multi-mode terminals.

Most of the previous research works relating to $A B C$ service either consider access network selection as static optimization problem which did not sufficiently address the dynamic nature of the network conditions obtaining dynamic QoS information 
such as available bandwidth altogether. So a new $4 \mathrm{G}$ architecture required to overcome the challenges of collecting real-time QoS information necessary for network selection. Moreover, the access network selection is triggered only when the service class or access point changes which undermined the varying network conditions otherwise.

The key limitation to ongoing research remains as no efficient way to obtain dynamic QoS information such as available bandwidth estimation required for network selection. Since 4G networks would be predominantly based on multimedia traffic, it is also important to consider the effects of selfsimilar traffic apparent in the IP-based core. These motivate us to estimate the available bandwidth of heterogeneous wireless networks as a dynamic QoS parameter for optimum network selection.

In our algorithm the available links must be ordered to determinate the best interfaces for the most important attribute. The simplest ordering technique is to make the network ordering based on one network parameter however it is not the best solution. The network selection algorithm must depend on the requested services. The ordering algorithm must be able to determine automatically the best interface and the interface order that fulfills the user's requirements. The parameters provided from network that the terminal uses for making the decision of multimedia applications are available bandwidth. Available bandwidth measurement is done by using online bandwidth estimation technique based on .net.

In this paper, available bandwidth estimation techniques that have been used for network selection in heterogeneous environment (WLAN \& WiMAX). In addition, a novel .net based bandwidth estimation technique is discussed, especially the characteristics required to make it an efficient method. The paper is organized as follows. Section II represents related work to network selection in heterogeneous environment; Section III describes the experimental set-up that has been built, whereas Section IV represents the design of available bandwidth estimation algorithm and its characteristics. Section V describes the simulation results. Finally, Section VI provides the conclusions and the future work in this direction.

\section{RELATED WORK:}

A number of researchers have proposed some network selection algorithms in the literature. A novel user preference oriented network selection algorithm (UPNS) was proposed, by combining entropy theory and fuzzy analytic hierarchy process algorithm (FAHP) with service level agreement (SLA) management model [4]. In [5], an effective access network selection algorithm for heterogeneous wireless networks was proposed that combines two Multi Attribute Decision Making (MADM) methods, the Analytic Hierarchy Process (AHP) method and the Total Order Preference by Similarity to the Ideal Solution (TOPSIS) method. In [6] they proposed an optimal distributed network selection scheme in heterogeneous wireless networks considering multimedia application layer QoS. QoS aware path selection scheme was proposed in [7], which estimates required bandwidth ratio based on the QoS requirements of target service and the SINR of each path. The proposed scheme could select the optimal path which can satisfy the QoS requirements among many heterogeneous wireless networks that change dynamically. They [8] introduced the muxer Global Arbitration Process (GAP) that balances application-oriented ratings of networks while optimizing the usage of communications resources and simultaneously meeting the application and user requirements.
Besides deciding which single network an application should use, GAP considers network bundles as well as a proactive delaying of data transfers as additional options to reach a global optimum for all applications running on the device. In [9] proposed a network selection algorithm which bases its decision on the estimated energy consumption. The proposed solution enables the multimedia stream to last longer while maintaining an acceptable user perceived quality by selecting the least power consuming network. Unlike previous works, this paper proposes a network selection criterion which uses an estimated available bandwidth as a dynamic parameter for real-time applications in order to select the best bandwidth efficient network.

\section{ARCHITECTURE OF HETEROGENEOUS NETWORKS:}

The general WLAN/WiMAX interworking environment consider for network selection in heterogeneous wireless network by using dynamic parameter as available bandwidth estimation is illustrated in figure 3. The necessary changes in both WLAN and WiMAX systems are rather limited as it will integrate both systems at the IP layer and relies on the IP protocol to handle mobility between access networks. The main characteristic of this architecture is to assume two overlapped cells of a Mobile WiMAX and a WLAN, where both cells are served by a Base Station (BS) and an Access Point (AP) respectively. The mobile terminal has dual interfaces: WiMAX and WLAN.

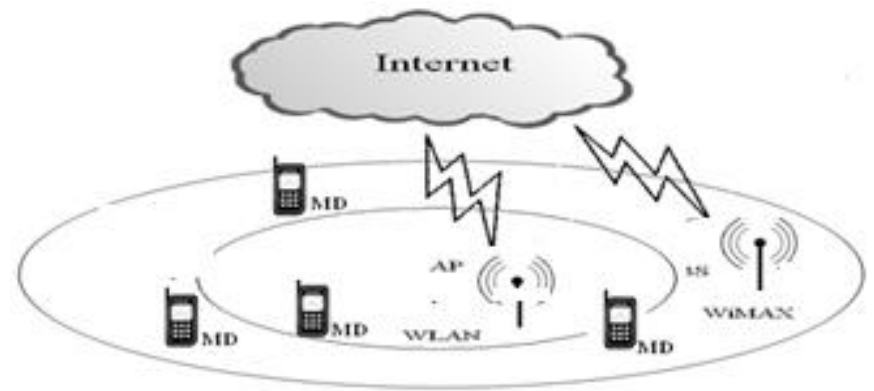

Figure 3: An integrated WiMAX and WLAN heterogeneous wireless network environment.

To select the always best connected (ABC) network in this environment, an available bandwidth estimation scheme is proposed. As the mobile device (cellular phone, laptop, palmtop etc.) moving one place to another according to the user preference the network is automatically selected. In the following section, available bandwidth estimation algorithm for network selection is presented.

\begin{tabular}{|c|c|c|}
\hline PROP & & AVAILABLE \\
\hline BANDWIDTH & & ESTIMATION \\
\hline $\begin{array}{l}\text { ALGORITHM } \\
\text { SELECTION: }\end{array}$ & FOR & NETWOR \\
\hline
\end{tabular}

Network performance depends on many factors. To understand the state of the network, modern operating systems support various performance monitoring mechanisms. Though useful, these features tend to drastically oversimplify the analysis of network performance. The performance of a wireless network connection depends in part on signal strength. Received signal strength (RSS) is a measurement of the power present in a received radio signal. In general, the received signal $r_{t}$ at the time instant $t$ can be expressed as [10] 
$r_{t}=a_{t} s_{t-\tau}+v_{t}$

Here, $s$ denotes the transmitted (pilot) signal waveforms, $a_{t}$ is the radio path attenuation, $\tau$ is the distance-dependent delay and $v_{t}$ is a noise component.

Between a computer and access point, the wireless signal strength in each direction determines the total amount of network bandwidth available along that connection. The available bandwidth (ABW) at a link is its unused capacity. Since, at any time, a link is either idle or transmitting packets at the maximum speed, the definition of the available bandwidth ought to look at the average unused bandwidth over some time interval T. Thus [11],

$\mathrm{A}_{\mathrm{i}}(\mathrm{t}, \mathrm{T})=\frac{1}{T} \int_{t}^{T+t}\left(c_{i}-\lambda_{i}(t)\right) d t$

Where $A_{i}(t, T)$ is the available bandwidth at link $i$ at time $t, C_{i}$ is the link's capacity, and $\lambda_{i}$ is its traffic. The available bandwidth along a path is the minimum available bandwidth of all traversed links.

A novel network selection scheme based on these performance parameters. The flow graph in figure 4 represents the logic of network selection based on available bandwidth estimation along with received signal strength (RSS) technique for finding always best connected criteria in heterogeneous environment for multimedia services. Because of multimode mobile device the user have opportunity to avail number of wireless network together. In this paper it is considered that of laptop has two interfaces capable to access WLAN and WiMAX networks. The selection of network depends upon the user preference that means on the application currently running. If the application is based on multimedia services, then the foremost requirement is bandwidth. So selection of the network depends on the network having more available bandwidth. To find out the more available bandwidth between the available networks (WLAN \& WiMAX) is calculated turn by turn.

$$
\text { No Yes }
$$

Figure 4: Represents the flow chart of available bandwidth estimation algorithm for network selection in heterogeneous wireless environment.

First of all select one of the available networks then download file of specific size from that particular network interface and note the time duration. The size of the file download required not to be large or nor to be small. Because if the size of file is large then it will take more time and more cost which is not preferable and if the size of the file is small then it can't accurately calculate the available bandwidth estimation. Download file size $=2397 \mathrm{~KB}$ is considered for testing purpose. Then calculate

Download bandwidth $=$ download file size $/$ time noted taken for downloading.

\{To calculate the download speed

speedtimer.Start()

readBytes(4095) As Byte

bytesread As Integer =

theResponse.GetResponseStream.Read(readBytes, 0, 4096)

nRead $+=$ bytesread

percent As Short $=(\mathrm{nRead} * 100) /$ length

If bytesread $=0$ Then Exit Do
writeStream.Write(readBytes, 0 , bytesread)

speedtimer.Stop ()

readings $+=1$

If readings $>=5$ Then

currentspeed $=20480 /$ (speedtimer.ElapsedMilliseconds / 1000)

speedtimer.Reset()

readings $=0$

End If

\}

The download bandwidth actually represents the available bandwidth of the current access interface. Then select the second interface and repeat the same process as discussed above. Then compare the calculated available bandwidth of both the interfaces i.e b1 (WLAN) and b2 (WiMAX) respectively.

$\{$ If $b 1=b 2$ Then

"Use any connection both have equal bandwidth"

$$
\begin{aligned}
& \text { ElseIf b1 > b2 Then } \\
& \text { "Select " connection with " bandwidth" b1 } \\
& \text { Else } \\
& \text { "Select " connection with " bandwidth" b2 } \\
& \text { End If ? }
\end{aligned}
$$

The interface has the high available bandwidth that is selected for the real time multimedia services as per user preference. In the following section, the practical implementation of the proposed algorithm is shown.

\section{IMPLEMENTATION OF AVAILABLE BANDWIDTH ESTIMATION ALOGRITHM FOR NETWORK SELECTION AND DISCUSSIONS:}

In this paper, WLAN (IEEE 802.11) and WiMAX (IEEE 802.16) based platform is constructed, as shown in Figure 3, the platform includes access point, base station and a number of dual mode mobile clients (laptops, cell phones or palm-tops ). A software platform for this architecture for network selection in this heterogeneous environment is developed by using .net. The software architecture of dual mode client is shown in figure 5 as follows.

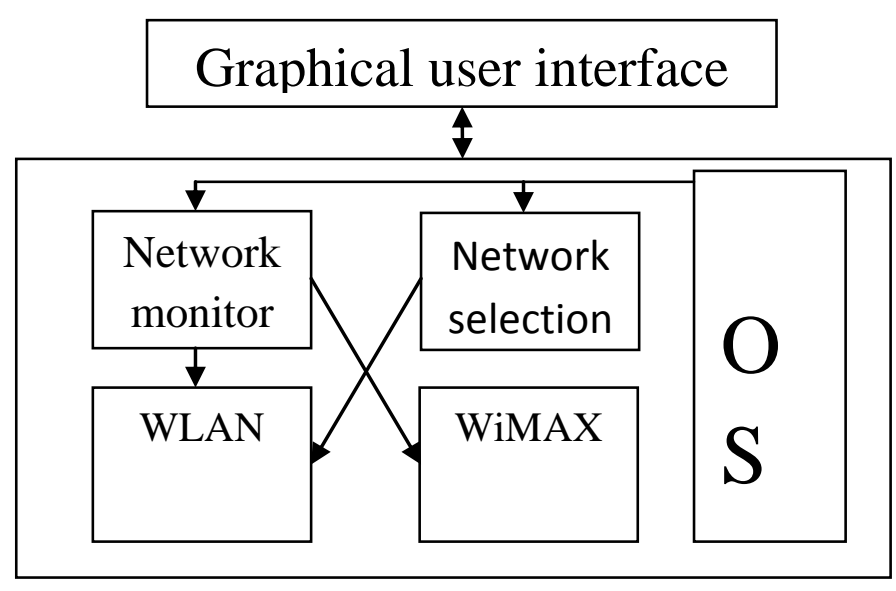

Figure 5: Software architecture of dual mode client.

To support seamless mobility across 802.11-based WLAN and 802.16 based WiMAX networks, development of mobile 
client software is done to manage multiple connections using 802.11 and WiMAX technologies. Such a client should also intelligently select and activate the ideal interface according to the network conditions.

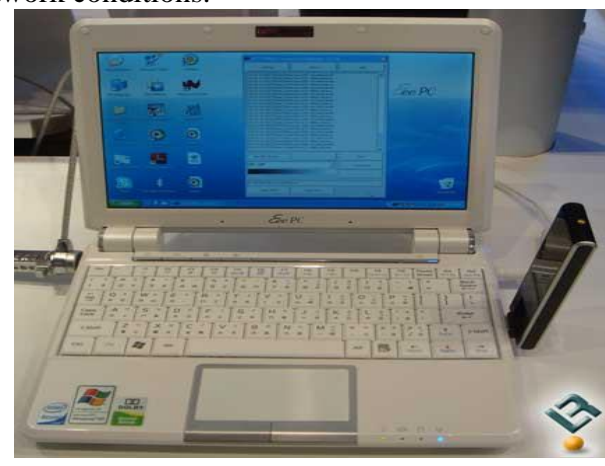

Figure 6: The dual mode mobile client [12]

However, there is no such client readily available. In this paper, developed of the dual mode client is done as shown in figure 6. Current implementation supports $802.11 \mathrm{~b} / \mathrm{g}$ (also known as WLAN) and WiMAX dongle as interfaces, and can easily be extended to other types of interface.

Dual mode client software provides support for automatic roaming across WLAN and WiMAX, which means that the client can monitor and automatically select WLAN or WiMAX network according to the available bandwidth estimation technique. The software system includes the following modules: a module to monitor and detect different wireless networks, a module to do network selection decision including to manage multiple connections.

Based on the integration architecture, a fully featured heterogeneous wireless test-bed comprised of WiMAX and WLAN network and software platform is deployed in the BSNL, Jalandhar. The software was implemented in a dualmode laptop having core 2 duo processer, T5800 @ 2.00 $\mathrm{GHz}, 3 \mathrm{~GB}$ of RAM. The software platform is net whose flow chart is shown in figure 2. The first selected interface is of WLAN of dual mode laptop and then second WiMAX interface is selected. The estimated available bandwidth at different instances with respective received signal strength of both interfaces of dual mode laptop is shown in table 1. By observing the table 1 it is concluded that the network having more available bandwidth is automatically selected for multimedia services. The received signal strength shows the presence or current status of the network in heterogeneous environment because available bandwidth estimation is only possible if network is present. But available bandwidth is not purely depended upon received signal strength (RSS) because high received signal strength didn't always mean for high available bandwidth because it also depends upon the number of user present at that time of estimation. This fact can also be observed from the table1.

Table 1: Estimated available bandwidth with received signal strength in heterogeneous environment of WiMAX and WLAN networks.

\begin{tabular}{|l|c|c|c|c|c|}
\hline $\begin{array}{l}\text { S.N } \\
\text { o }\end{array}$ & \multicolumn{2}{|c|}{ WLAN } & \multicolumn{2}{c|}{ WiMAX } & $\begin{array}{c}\text { Network } \\
\text { Selection }\end{array}$ \\
\hline & $\begin{array}{c}\text { RSS } \\
(\mathbf{d B})\end{array}$ & $\begin{array}{c}\text { BW } \\
(\mathbf{K b} / \mathbf{s})\end{array}$ & $\begin{array}{c}\text { RSS } \\
(\mathbf{d B})\end{array}$ & $\begin{array}{c}\text { BW } \\
(\mathbf{K b} / \mathbf{s})\end{array}$ & $\begin{array}{c}\text { Selected } \\
\text { networks }\end{array}$ \\
\hline 1. & -45.0 & 68.03 & -54.76 & 2.33 & WLAN \\
\hline 2. & -40.0 & 77.82 & -70.93 & 149.25 & WiMAX \\
\hline 3. & -41.0 & 53.19 & -54.65 & 155.04 & WiMAX \\
\hline 4. & -44.0 & 2.34 & -57.78 & 148.15 & WiMAX \\
\hline
\end{tabular}

By comparing the both network's available bandwidth, the WiMAX/WLAN network is selected for multimedia services as per user's preference as shown in figure 7 .

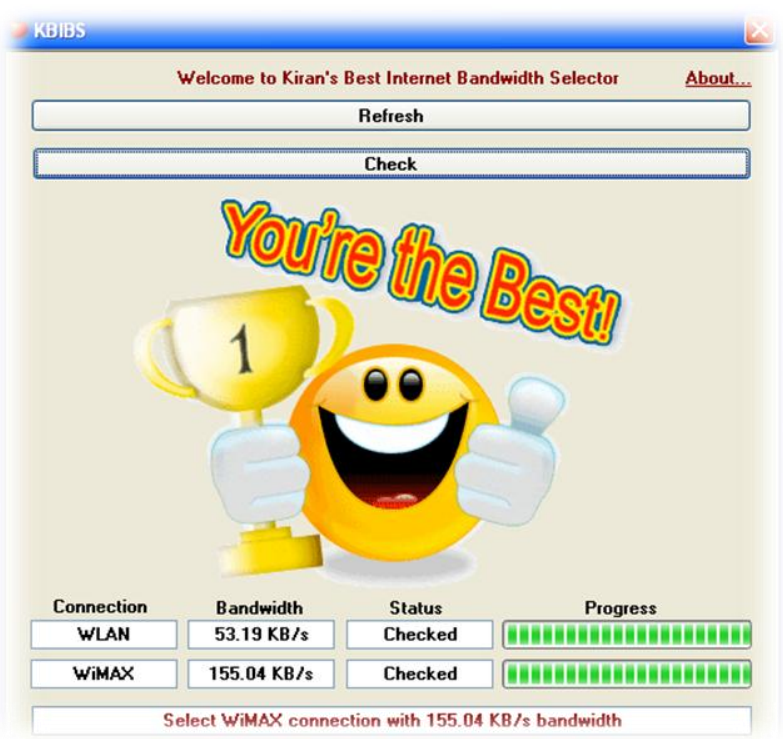

Figure 7: User interface for network selection algorithm based on available bandwidth estimation in heterogeneous environment of WiMAX and WLAN network.

All the real implementation and experimental results proves the efficiency and effectiveness of available bandwidth estimation algorithm because in this algorithm measure available bandwidth by taking average of readings so that it will show more accurate estimated bandwidth rather than showing the only one instance estimated available bandwidth. It can be considered as a practical solution for seamless communication among heterogeneous wireless networks by accurate network selection for multimedia services.

\section{CONCLUSION AND FUTURE WORK:}

In this paper, described the design and a real world implementation of proposed algorithm, for ubiquitous Internet access in heterogeneous environment of WLAN and WiMAX. Both hardware and software systems are developed for integrating WLAN and WiMAX networks. Practical implementation has been deployed in BSNL Office, Jalandhar. Through extensive experiments and testing, dynamic access network selection algorithm based on dynamic parameter i.e available bandwidth has been proved to be effective and efficient for real-world applications. Depending upon the availability of the network the selection is performed between WLAN and WiMAX networks by available bandwidth estimation whereas availability of the network is confirmed by measuring received signal strength (RSS). Proposed work provides insights into practical deployment of heterogeneous wireless network and is valuable to future researches. Also, it is important to notice that although proposed algorithm consists of WLAN and WiMAX networks, but it can be easily applied to heterogeneous environments composed of other wireless networks, e.g. 3G networks. 
In the future, extensive performance testing and analysis to provide more quantitative results can be done. Proposed algorithm can be improved in different aspects viz. delay due to calculation of available bandwidth and cost of downloading a specific size of file.

\section{REFERENCES:}

[1] Eng Hwee Ong, Jamil Y. Khan, "Dynamic Access Network Selection with QoS Parameters Estimation: A Step Closer to ABC" IEEE VTC 2008-Spring, 2671-2676.

[2] "WiMAX and WLAN Together: Deployment Models and User Scenarios" White paper Co-authored by Motorola and Intel, 2007, pp 1-10

[3] Chen Yiping and Yang Yuhang, "A new 4g architecture providing multimode terminals always best connected services". IEEE Wireless Communications, 14(2):36-41, April 2007.

[4] Xiaoli Ai, Wenan Zhou, Bing Xie, and Junde Song, "Network Selection Issue in Heterogeneous Wireless Environment", Wireless Communications and Networking Conference (WCNC), 2010 IEEE, pp 1-6, 18-21 April 2010

[5] Aggeliki Sgora, Dimitrios D. Vergados, Periklis Chatzimisios, "An Access Network Selection Algorithm for Heterogeneous Wireless Environments", Computers and Communications (ISCC), 2010 IEEE Symposium on 22-25 June 2010, pp 890-892

[6] Pengbo Si, Hong Ji, F. Richard Yu, (2010), "Optimal network selection in heterogeneous wireless multimedia networks", Wireless Networks, Volume 16, pp: $1277-$ 1288
[7] Shin-Hun Kang, Jae-Hyun Kim, "QoS-aware path selection for multi-homed mobile terminals in heterogeneous wireless networks", Consumer Communications and Networking Conference (CCNC), 7th IEEE, 9-12 Jan. 2010, pp 1-2

[8] Sven Lahde, Martin Wegner and Lars Wolf, "Efficient Network Selection in Heterogeneous Communication Scenarios using Arbitration" World of Wireless Mobile and Multimedia Networks (WoWMoM), 2010 IEEE International Symposium on 4-17 June 2010, pp 1 - 6.

[9] Ramona Trestian, Olga Ormond, Gabriel-Miro Muntean, "Power-Friendly Access Network Selection Strategy for Heterogeneous Wireless Multimedia Networks", Broadband Multimedia Systems and Broadcasting (BMSB), 2010 IEEE International Symposium on 24-26 March 2010, pp $1-5$.

[10] Mussa Bshara, Umut Orguner, "Fingerprinting Localization in Wireless Networks Based on ReceivedSignal-Strength Measurements: A Case Study on WiMAX Networks" IEEE Transactions On Vehicular Technology, VOL. 59, NO. 1, pp 283-294, January 2010

[11] Jacob Strauss, Dina Katabi, Frans Kaashoek, "A Measurement Study of Available Bandwidth Estimation Tools" IMC'03, October 27-29, 2003

[12]http://www.legitreviews.com/images/news/2008/eeepc_w imax_dongle.jpg 